\title{
Analysis on Rehabilitation Status of Sports Injuries
}

\author{
Ming $L i^{1, a}$ \\ ${ }^{1}$ Dalian Institute of Science and Technology, Dalian 116052, China \\ a liming02030206@163.com
}

\begin{abstract}
Keywords: Sports injuries, Rehabilitation, Intervention
Abstract. The various injuries that occur in the process of physical fitness and competitive sports are collectively referred to as sports injuries. At present, sports injuries are still the most formidable enemy in the career of athletes and non-athletes. Many athletes who have achieved excellent results often are forced to complete their career prematurely because of varying degree of injuries. A factor that prolongs the sports life is to avoid the occurrence of sports injuries accurately and effectively. What kind of methods can be used to actively interfere with sports injuries more effectively to make the injured recover completely, return to the peak in a shorter time and return to the sports field becomes a key link in the development of competitive sports and mass sports to this day. In this paper, the status quo of rehabilitation of sports injuries is discussed through consulting literature to provide suggestions for the recovery of sports injuries.
\end{abstract}

\section{Introduction}

At present, sports injuries can be seen everywhere and vary in variety. How to deal with sports injuries in a short period of time and correctly handle and effectively intervene is particularly important for rehabilitation after sports injuries ${ }^{[1]}$. Nowadays, a greater number of people have more or less certain misunderstandings about the rehabilitation of sports injuries and their processes, which may cause more serious sports injuries ${ }^{[2]}$. Therefore, it is significant to understand how to deal with common sports injuries and perform post-traumatic rehabilitation.

\section{Basic goals of the rehabilitation process for sports injuries}

The main task and ultimate goal of sports injury rehabilitation is to enable the injured to recover to the original or even better level of exercise and competitive state, and in particular to shorten the rehabilitation cycle of the injury as much as possible under the conditions permitting ${ }^{[3]}$. In the entire process of sports injury rehabilitation, not only should the pains relief of the injured be targeted, but also the psychological health of the injured should be ensured, so that the injured can actively cooperate with the treatment and the body can be in a good condition and healthy state. Currently, the rehabilitation of sports injuries need to achieve the following goals.

\subsection{General Physical Training}

Physical training includes general physical training, specialized physical training and special physical training ${ }^{[4]}$. In general, when the sports injury occurs, the training will be paused for the first time. The training here mainly refers to specialized physical training and special physical training, and general physical training should continue. If all kinds of physical training are stopped immediately after the sports injury appears, it will be beneficial to recover the damage from a certain point of view. However, after the injury is recovered, certain physical qualities such as speed, strength, etc. cannot be returned to the previous level before the injury immediately, and the resumption of physical fitness training will take much longer, which does not meet our correct understanding of sports injury rehabilitation ${ }^{[5]}$. The correct approach is to try to ensure general physical training of the injured part and its surrounding areas without causing pain at the injury. 


\subsection{Alleviate the Corresponding Pain}

During the rehabilitation period, the injured part is often accompanied by varying degree of pain. Pain will not only affect the rehabilitation training in this period to a certain extent, but will also affect the mental state of the injured, such as impatience, anxiety, irritability and other emotions, thus affecting the progress of rehabilitation and extending the time required for the rehabilitation process, even causing new injuries to extremists. The relief of pain is to be prioritized after the occurrence of sports injuries. The PRICE principles need to be followed, namely, protection, immobilization, ice compression, compression bandage and evaluation, of which ice compression is a critical part.

\subsection{Maintain Cardiopulmonary Function}

During the injury, if the training is stopped directly, the cardiopulmonary function of the injured will be reduced to some extent. Because the injured had participated in exercise training before the injury, the cardiopulmonary function had reached a certain level. To maintain this level, appropriate training should be performed without affecting the injured part, and the prerequisite is certainly to cause injury pain. It is better to say that if the upper limbs are damaged, then more training of the lower extremities can be preformed during the rehabilitation training to maintain the level of the cardiopulmonary function unchanged or even improved.

\subsection{Recover joint movement}

Many sports injured occur near the joints or affect the joints. If the joint is forced to be fixed at a certain angle while dealing with sports injuries, then the joint shall be unfixed after a certain period of time without causing any pain, and appropriate amount of joint motion training shall be performed. Otherwise, additional training is required to recover to the original scope of activities if the joint remains fixed for a long period of time, which is contrary to the rehabilitation we are pursuing.

\subsection{Recover Muscle Strength and Endurance}

Recovering muscle strength and endurance during the recovery cycle of sports injuries is an extremely important part of the entire rehabilitation process. At present, isometric training and isokinetic training are two commonly used methods that can promote muscle training and effectively ensure muscles to receive appropriate stimulation. However, in the course of this exercise, there will generally be some degree of pain and discomfort. It is necessary to ensure that the athletes are trained in a painless state to the maximum extent possible to avoid secondary injuries.

\subsection{Recovery of Body Motion Perception, Muscle Movement Sensation and Neuromuscular adjustment}

In the injury recovery cycle, it is necessary to focus on adaptive recovering training of the injured person's own motion perception and muscle movement sensation, and in particular, to use neuromuscular adjustment to maintain a better level of his or her corresponding motor skills. In this process, the corresponding training of spatial sense shall be used to improve the corresponding skills, and to enable the injured to form an inertia.

\section{Psychological Intervention Treatment During Recovery Period of Sports Injuries}

During the injury period, besides the physical pain and obstacles, the injured will also have varying degree of psychological changes. The more common ones include impatience, anxiety, irritability, suspicion, indifference, panic, self-abandonment and other emotions. How to correctly handle these emotions of the injured is also very important for the rehabilitation of sports injuries. While performing rehabilitation training, it is necessary to try to adjust the abnormal emotions of the injured, or to appease or motivate the injured to maintain a good attitude, which is conducive to the rehabilitation training and physical recovery. Do not try to use extreme or negative words to stimulate 
the injured to undergo rehabilitation training, which tends to backfire and leave a psychological shadow on the patient, hindering recovery from sports injuries.

\section{Analysis on Rehabilitation Exercise Method}

\subsection{Rehabilitation Exercise Method for Different Types of Muscle Contraction}

\subsubsection{Rehabilitation exercise method of concentric contraction}

Concentric contraction, also known as shortened contraction, refers to the contraction of a shortened length when the muscle contracts. During the concentric contraction, the length of the muscle is shortened, the start and end point are close to each other, thereby causing the body to move. Concentric contraction is the form of contraction of active skeletal muscle, and is also the most commonly used training method in rehabilitation.

\subsubsection{Rehabilitation training of isometric contraction}

Isometric contraction, also known as static contraction, refers to that the length of muscle remains unchanged when it contracts. When the muscle is isometrically contracted, it cannot overcome against resistance to have mechanical work because its length remain unchanged.

\subsubsection{Rehabilitation exercise of centrifugal contraction}

Centrifugal contraction is a contraction that is stretched while muscles contract and produce tension. In theory, centrifugal contraction is the most effective way of muscle training, but at present, its application is far less than the concentric contraction in the rehabilitation training. Centrifugal contraction is more recommended for rehabilitation training, and helps to shorten the time of rehabilitation treatment.

\subsection{Power selection in rehabilitation exercises}

During the muscle training in rehabilitation treatment, the selection of resistance is often involved. In general, in the initial stage of recovery or the recovery process requiring only the motion of joints, it is only necessary to overcome the resistance. As rehabilitation training progresses, appropriate resistance is usually required to achieve the desired effect. The selection of resistance should follow the corresponding relationship between the percentage of 1RM and the number of times (see Table 1). $1 \mathrm{RM}$ refers to the amount of resistance that muscles can resist when they only contract once. Generally speaking, 65\% 75\% of 1RM will be selected for resistance training, and the corresponding 12 8 times is the most appropriate, which will also leading to the most obvious effect. The selection of excessive resistance will cause unnecessary damage to the muscles, and if two small resistance is selected, the corresponding recovery time will be longer.

Table 1. 1RM Percentage and Times

\begin{tabular}{cc}
\hline 1RM Percentage (\%) & $\begin{array}{c}\text { Order of Times of repetition } \\
\text { /number of classes }\end{array}$ \\
\hline $90 \sim 95$ & $1 \sim 2$ \\
\hline $85 \sim 90$ & $3 \sim 4$ \\
\hline $80 \sim 85$ & $4 \sim 6$ \\
\hline $75 \sim 80$ & $6 \sim 8$ \\
\hline $70 \sim 75$ & $8 \sim 10$ \\
\hline $65 \sim 70$ & $10 \sim 12$ \\
\hline
\end{tabular}

\subsection{Rehabilitation Process Under Passive Exercise}

Passive exercise refers to the process in which the curer applies an external force to make the patient perform activities without the active force, that is, the muscle does not actively contract. It is usually used for passive joint mobility training, and is often adopted in the early stage of rehabilitation. After a period of rehabilitation training, it is better to use a power-assisted exercise or active training instead of passive exercises, otherwise, the rehabilitation will be slowed after muscles and joints recover to a certain degree. 


\subsection{Rehabilitation Process Under the Power-assisted Exercise}

In this basic process, patients cannot rely solely on their own strength to exercise on their own, and they need some help or exercise with the help of others. The power-assisted exercise is suitable for periods when passive exercise reaches a certain degree and active training is not available. The power-assisted exercise should gradually increase the power for the exercisers step by step to enable then to bear a certain amount of load slowly. In the process, the corresponding assistance can not only come from different sizes of devices, but also from others and themselves.

\subsubsection{Rehabilitation Process Under Active Exercise}

The rehabilitation process under active exercise is aimed more at enhancing the muscles' strength, so as to seek a large movement of the joints. Active exercise not only plays a decisive role in the recovery of muscle strength, but also has a beneficial effect on the cardiopulmonary function. At this stage, the injured do not need to relay on any device or others, and they can carry out the rehabilitation exercise by themselves. They can even perform appropriate resistance exercise. However, the extent to which the injured can perform depends on the individual and must not be impatient and rushed.

\subsubsection{Rehabilitation Process Under Resistance Exercise}

The rehabilitation training under certain resistance can better increase the strength of muscles around the injured part of the rehabilitation person, and it is of great significance to the recovery of the physical function of the rehabilitation person. The specific practice of resistance exercise is that the exerciser should increase the corresponding exercise load under the premise of labor saving according to the corresponding principles of physical mechanics. In particular, certain rules shall be based to ensure that the corresponding resistance can be flexibly adjusted during the entire exercise process, and particularly ensure the resistance during the start and end of the activity to be kept under a relatively small state, so that the exercisers are subject to greater resistance in the middle of the process.

\section{Conclusion}

From the above analysis, it can be seen that at present, in the rehabilitation process of injuries, it is necessary to choose the rehabilitation measures and methods that are more suitable for the injured in the light of their actual situation. Moreover, in thee process of treatment and recovery, the possible adverse effects on different parts of the body caused by the injury shall be intervened, so as to actively and effectively intervene in the recovery of the injured. In addition, the entire rehabilitation process should be a step-by-step process, which should not be rushed, and shall ensure athletes to recover and restore to health.

\section{References}

[1] Wei Yi, Prevention and Rehabilitation Training for Sports Injuries of Public Security College Students, Tonghua Normal University Report, 2008, 29(12).

[2] Wei Yi. Investigation and Analysis of Sports Injuries of Public Security College Students. Tonghua Normal University Report, 2008, 29(8).

[3] Wei Yi. Investigation and Analysis of Sports Injuries of Public Security College Students. Journal of Henan Institute of Technology (Science Edition). 2009, 37(3).

[4] Zhang Xiaohui, Epidemiological Investigation of Actual Combat training Injuries of Police College Students, China Clinical Rehabilitation, 2006, 10(20).

[5] Xie Yong, Hou Lerong, Thoughts on Sports Injury Rehabilitation System Prepared for Beijing Olympics ,Journal of Chinese Sports School,2008, 34(3). 